\title{
Role of Myocardial Properties and Pacing Lead Location on ECG in Personalized Paced Heart Models
}

\author{
Konstantin S Ushenin ${ }^{1,2}$, Arseniy Dokuchaev ${ }^{2}$, Sonya M Magomedova ${ }^{4}$, Oleg V Sopov ${ }^{4}$, \\ Vitaly V Kalinin ${ }^{5}$, Olga Solovyova ${ }^{1,2,3}$ \\ ${ }^{1}$ Ural Federal University, Ekaterinburg, Russia \\ ${ }^{2}$ Institute of Immunology and Physiology Ural Branch of RAS, Ekaterinburg, Russia \\ ${ }^{3}$ Institute of Mathematics and Mechanics Ural Branch of RAS, Ekaterinburg, Russia \\ ${ }^{4}$ Bakoulev Scientific Center for Cardiovascular Surgery, Moscow, Russia \\ ${ }^{5}$ EP Solutions SA, Yverdon-les-Bains, Switzerland
}

\begin{abstract}
Personalised cardiac models were built from the computed tomography imaging data for two patients with implanted cardiac resynchronisation therapy devices. The cardiac models comprised a biventricular model of myocardial electrophysiology coupled with a model of the torso to simulate the body surface potential map. The models were verified against electrocardiogams (ECG) recorded in the patients from 240 leads on the body surface under left ventricular pacing. The simulated ECG demonstrated a significant sensitivity to the myocardial anisotropy and location of the pacing electrode tip in the models. An apicobasal cellular heterogeneity was shown to be less significant for the ECG pattern at the pacedventricle activation than that showed earlier by Keller and co-authors (2012) for the normal activation sequence.
\end{abstract}

\section{Introduction}

In the earlier modelling study [1], Keller and co-authors found that cellular electrophysiological heterogeneity and anisotropy in the ventricular myocardium significantly influence the electrocardiogram (ECG) produced under normal cardiac activation. In the present study, we used personalised computational models to investigate how myocardial anisotropy, heterogeneity and pacing lead location may affect ECG in the case of ventricular pacing from a point source.

This problem is significant for several reasons. An abnormal activation of ventricles from a point source may occur in cases of electrical pacing or premature ventricular beats. Particularly, our simulations may be useful for understanding the relationships between myocardial properties and the success of cardiac resynchronisation therapy (CRT). Also, such simulations may be used to deter- mine conditions when premature ventricular beats initiate re-entry or ventricle fibrillation. In addition, the role of cardiac anisotropy and heterogeneities in the inverse problem of electrocardiograpy is not fully understood.

\section{Methods}

\subsection{Clinical data}

In this pilot study, two patients (see Table 1 for details on the Case 1 and Case 2 patients) with cardiomyopathy and implanted CRT devices were examined. ECG data were recorded under a left ventricular (LV) pacing mode provided by a coronary sinus pacing electrode and used to validate patient-specific models of cardiac electrical activity. The patients underwent computed tomography (CT) and noninvasive body surface ECG mapping in 12 standards and 240 unipolar ECG leads (Amycard 01C, EP solutions SA) [2]. 3D anatomical models of the torso and the right and left ventricles of the heart with a precise position of the pacing electrode were derived from the CT data using Amycard $01 \mathrm{C}$ system software. The tetrahedral finite element meshes of the ventricles and torso were then generated by GMSH software to calculate the electrical activity in the ventricular myocardium and simulate ECG signals in the same torso points as recorded.

\begin{tabular}{l|cc}
$\#$ & Case 1 & Case 2 \\
\hline Gender & Female & Male \\
Age & 67 & 56 \\
QRS Duration & 208 & 230 \\
Main Diagnosis & Arrhythmogenic & Hypertrophic \\
& Cardiomyopathy & Cardiomyopathy
\end{tabular}

Table 1: Patient cases 


\subsection{Electrophysiology}

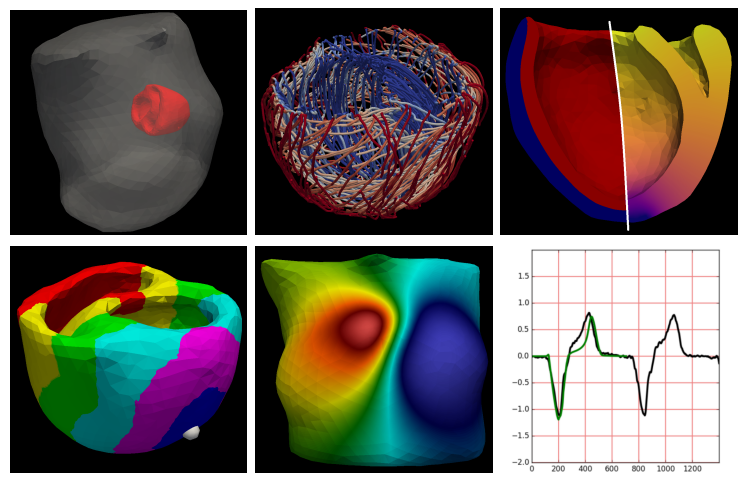

Figure 1: Personalised cardiac model for Case 1 patient. From top to bottom, left to right: model of the torso and the heart geometry; fiber orientations; transmural and apicobasal cellular heterogeneity; activation map (white point shows an activation source); body surface potential map at the time of QRS peak; a simulated II standard lead ECG signal (green) against recorded ECG (black).

The patient-specific model comprised a biventricular heart model and a homogeneous torso model (Fig. 1). Electophysiology of the myocardium was calculated using a bidomain model with an ionic model of human ventricular cardiomyocites [3] and strong heart-torso coupling [4]. A fiber orientation field was mapped to the model using a rule-based approach [1] (Fig. 1). The conduction anisotropy ratio along and across the fibers was 9:1. A transmural cellular heterogeneity in the ventricles was simulated by two equal-width layers of the wall consisting of the subepicardial- and subendocardial-type cellular models [3] (Fig. 1). An apicobasal cellular heterogeneity was simulated using a linear gradient of the conductivity $g_{K s}$ for the slow potassium current as described in [1] (Fig. 1). The membrane capacitance and the ratio of electrodiffusion coefficients were taken from [3]. A surface-tovolume ratio was fitted to the QRS amplitude and duration for the standard lead II signal (Fig. 1). The myocardial stimulation in the model was applied at the location of the pacing electrode tip on the subepicardial surface of the LV free wall derived from the patient CT (Fig. 1). Simulations of cardiac electrophysiology were run with the Chaste software [5].

We call the patient-specific models with a basic set of parameters as 'reference models', which output was compared with tested models with certain parameters varied. For both patient cases, the reference models produced a gradient of action potential duration from $268 \mathrm{~ms}$ at the base to $330 \mathrm{~ms}$ at the apex and a conduction velocity in the myocardial tissue equal to $0.6 \frac{\mathrm{m}}{\mathrm{s}}$ along and $0.2 \frac{\mathrm{m}}{\mathrm{s}}$ transverse the fibers, which are in the physiological range.

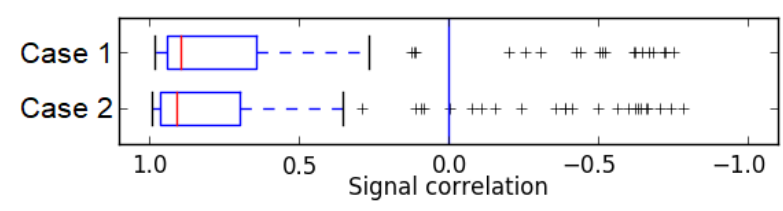

Figure 2: Distribution of the signal correlation metric.

\subsection{Model validation}

Three metrics were used to compare the simulated results with the clinical data:

$$
\begin{aligned}
S C(x, y) & =\frac{\sum_{i=0}^{T} x_{t} y_{t}}{\sqrt{\sum_{i=0}^{T} x_{t} \sum_{i=0}^{T} y_{t}}} \\
E D(x, y) & =\sqrt{\sum_{t=0}^{T}\left(x_{t}-y_{t}\right)^{2}} \\
Z M_{\Delta t, \mu}(x, y) & =\min _{\Delta t, \mu} \sqrt{\sum_{t=0}^{T}\left(x_{t}-\operatorname{zoom}\left(y_{t+\Delta t}, \mu\right)\right)^{2}} .
\end{aligned}
$$

Here, $x_{t}$ is an experimental time-discrete ECG signal recorded from a torso surface lead and $y_{t}$ is a simulated ECG signal generated by the model at the same location.

The signal correlation (SC) metric is useful for checking the co-orientation of the QRS complex and T-wave in the signals [1]. However, this metric is not quite sensitive to amplitude differences. To account for differences in the signal shape and amplitude, we used the discrete Euclidean distance (ED).

The reference models used generalised (based on 'population') values for conductivity, membrane capacitance and surface-to-volume ratio, but these myocardial properties are patient specific and vary between subjects. Here, we proposed a Zoom Metric (ZM), which accounts for a small time shift $\Delta t \in[+20,-20] \mathrm{ms}$ and time-scaling zoom $\left(y_{t}, \mu\right)$ with factor $\mu \in[0.9,1.1]$ of the simulated signal to minimise the distance from the experimental signal. The ZM was implemented using the scipy module in Python. The ZM is able to minimise small variations in QRS and the T-wave width due to varying individual conduction velocity in the real myocardium, but the $\mathrm{ZM}$ is sensitive to other ECG parameters (e.g., amplitude and orientation of ECG peaks, etc).

\section{Results}

First, the reference model for each patient was validated against the clinical ECG signals recorded from 240 electrodes on the body surface of the patient (Fig. 2). Here, we used the SC metric as suggested in [1]. The distribution of the SC had a median close to 1 and a variance of 


\begin{tabular}{|c|c|c|c|c|}
\hline & \multicolumn{2}{|c|}{ Euclidean distance } & \multicolumn{2}{|c|}{ Zoom metric } \\
\hline & Case 1 & Case 2 & Case 1 & Case 2 \\
\hline $\begin{array}{l}\text { Reference model (conventional anisotropy ratio } 9: 1, \\
\text { apicobasal heterogeneity, subepicardial pacing lead location) }\end{array}$ & $100 \%$ & $100 \%$ & $100 \%$ & $100 \%$ \\
\hline Isotrophy (conductivity ratio $1: 1$ ) & $140 \%$ & $150 \%$ & $114 \%$ & $116 \%$ \\
\hline Moderate anisotropy (conductivity ratio $4: 1$ ) & $90 \%$ & $97 \%$ & $88 \%$ & $92 \%$ \\
\hline High anisotropy (conductivity ratio $16: 1$ ) & $107 \%$ & $104 \%$ & $105 \%$ & $104 \%$ \\
\hline No heterogeniety & $108 \%$ & $106 \%$ & $101 \%$ & $102 \%$ \\
\hline Varied pacing lead location & $99 \%-118 \%$ & - & $98 \%-118 \%$ & - \\
\hline Subendocardial pacing lead location & $107 \%$ & - & $106 \%$ & - \\
\hline
\end{tabular}

Table 2: Effect of model parameters on simulated ECG metrics.

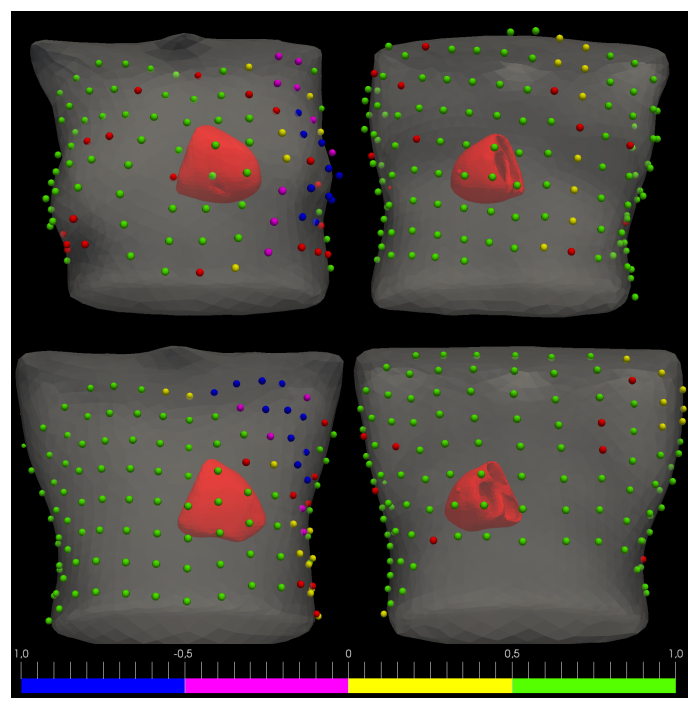

Figure 3: The ECG signal correlation (SC) metric at the torso surface electrodes mapped to Case 1 (top) and Case 2 (bottom). The colour schematic ranges from green for the highly correlated signals to blue for the inversely correlated signals red colour indicates disconnected electrodes.

about 0.4 for each patient (Fig. 2). The SCs for more than $50 \%$ of the electrodes were inside the box-range from 0.65 to 0.9 , and more than $80 \%$ of the simulated ECG showed $S C>0.5$, which indicates good qualitative similarity between the simulated and the experimental ECG (Fig. 3). Electrodes with SC metrics lying outside the box-plot 'whiskers' were mostly located on the left side of the chest. Here, the simulated ECG with the negative SC showed inverted QRS and T-wave polarity as compared to the clinical data.

To evaluate the effect of myocardial anisotropy, we set the fixed value of the conductivity along the myocardial fibers and ran both patient-specific models with varying ratios of conductivity coefficients along to across the fibers: 1:1 (isotropic tissue), 4:1 (moderate anisotropy), 9:1 (reference anisotropy ratio, which widely used to model normal myocardial tissue, e.g., [6]) and 16:1 (high anisotropy). The anisotropy variations resulted in the ratio of the con- ductance velocity along to across the fibers: 1:1, 2:1, 3:1 and $4: 1$, respectively. The values of the ED metric and the $\mathrm{ZM}$ metric in relation to the reference models are presented in Table 2.

To evaluate the role of the cellular apicobasal heterogeneity, we excluded the heterogeneity and compared the clinical ECG data with the simulated data (Table 2). Exclusion of the heterogeneity from the model did not lead to a change in T-wave polarity in either of the electrodes. Moreover, the ZM metric did not indicate a significant difference between the simulations.

In the next series of experiments, we investigated the effect of the pacing lead location on the ECG in the Case 1 model (Table 2). The pacing site was randomly shifted around the reference location in $0.5-3 \mathrm{~cm}$. Models were calculated with 18 different pacing sites. All the tested pacing sites were projected to the tangent plane in the reference location. Subsequently, the ED metric was interpolated by RBF interpolation to build a map of the metric depending on the pacing site displacement (Fig. 4). In addition, we examined the effect of a displacement of the reference pacing site from the subepicardial to the subendocardial surface of the left ventricle.

We found that the ED-metric map is a convex function. The minimum was located at a distance less than $1 \mathrm{~cm}$ from the reference point, which is comparable to the error in the visual identification of the point source location derived from the CT. A similar map was built for the ZM, which also showed a minimum located less than $1 \mathrm{~cm}$ from the reference point.

\section{Discussion}

The distribution of the SC metric was similar to the results of Keller and co-workers [1], which simulated the normal activation sequence of the ventricles. We assume that the quality of our simulations is suitable for future studies.

Myocardial anisotropy was shown to be an essential model property for the generation of adequate ECG signals. In terms of the ED metric and the $\mathrm{ZM}$, the great- 
est distance of the simulated ECG from the real signal was shown in the isotropic myocardium model (conductivity ratio $1: 1$ ), which was not eliminated by signal timezooming. The simulated signals closest to the clinical data were produced at the moderate anisotropy (conductivity ratio $4: 1$ ). This ratio agrees with data obtained in human and other mammals [7]. The sensitivity of the simulated ECG to the parameters of myocardial anisotropy suggests that it is possible to identify this model parameter on the basis of the experimental ECG data.

A recent paper by Keller and co-workers [1] showed that apicobasal cellular heterogeneity in ventricles is essential for the morphology of the T-wave under a normal myocardial activation sequence. By contrast, our simulations showed no effect of cellular heterogeneity on the Twave polarity in the case of ventricular pacing from the left ventricular site. This result indicates that activation pattern is important for T-wave properties.

The last series of our experiments showed that the ECG was significantly sensitive to the location of the pacing electrode tip. The shift of the pacing site from the reference location led to an almost gradual increase in the distance between the simulated and experimental ECGs. The effect was quantitatively more expressed than the effect of cellular heterogeneity, while comparable with the effect of the change in the anisotropy ratio. The models also showed an appreciable change in the ECG metrics when the pacing site was moved from the epicardial to the endocardial ventricular surface. The results suggest that the myocardial electrical activity has rather high sensitivity (reflected in the ECG change) to the particular pacing lead location.

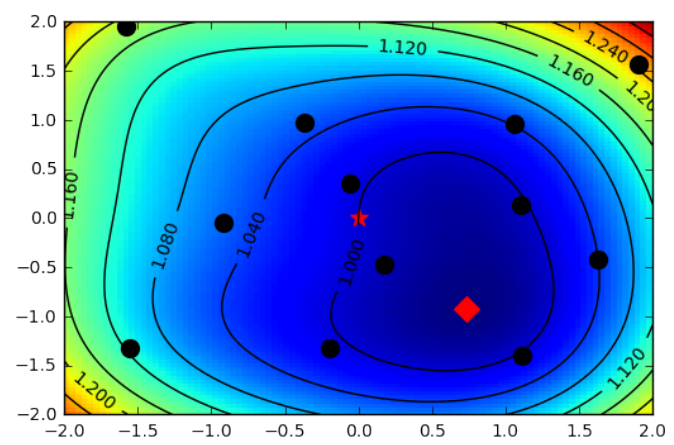

Figure 4: Euclidian distance (ED) map for simulated ECG depending on the pacing site distance from the reference location (marked by the red star). Black points show the locations of tested pacing sites in the model. Red diamond indicates position of the pacing site with minimum ED metric.

\section{Conclusion}

In this study, based on clinical data, we built patientspecific electrophysiological cardiac models paced from the LV epicardial site.
We showed that myocardial anisotropy plays an essential role in the propagation of the excitation wave and the genesis of the ECG. At the same time, apicobasal heterogeneity is less significant for the ECG pattern in the paced heart than for the normal activation sequence.

We found a significant sensitivity of the model to the pacing lead location, in particular to the displacement of the pacing site from the epi- to the endocardial LV surface.

Our results support the use of personalised cardiac computation models for the planning of CRT.

\section{Acknowledgements}

This study was supported by the RAS Presidium Programme I.33П, and Government of the Russian Federation (agreement 02.A03.21.0006). We used the computational clusters of Ural Federal University and "URAN" of Institute of Mathematics and Mechanics (Ekaterinburg).

\section{References}

[1] Keller DU, Weiss DL, Dossel O, Seemann G. Influence of $I_{K s}$ heterogeneities on the genesis of the t-wave: A computational evaluation. IEEE Transactions on Biomedical Engineering 2012;59(2):311-322.

[2] Revishvili AS, Wissner E, Lebedev DS, Lemes C, Deiss S, Metzner A, Kalinin VV, Sopov OV, Labartkava EZ, Kalinin $\mathrm{AV}$, et al. Validation of the mapping accuracy of a novel noninvasive epicardial and endocardial electrophysiology system. Europace 2015; euu339.

[3] ten Tusscher KH, Panfilov AV. Alternans and spiral breakup in a human ventricular tissue model. American Journal of Physiology Heart and Circulatory Physiology 2006; 291(3):H1088-H1100.

[4] Boulakia M, Cazeau S, Fernández MA, Gerbeau JF, Zemzemi N. Mathematical modeling of electrocardiograms: a numerical study. Annals of biomedical engineering 2010; 38(3):1071-1097.

[5] Mirams GR, Arthurs CJ, Bernabeu MO, Bordas R, Cooper J, Corrias A, Davit Y, Dunn SJ, Fletcher AG, Harvey DG, et al. Chaste: an open source c++ library for computational physiology and biology. PLoS Comput Biol 2013;9(3):e1002970.

[6] Keller DU, Jarrousse O, Fritz T, Ley S, Dossel O, Seemann G. Impact of physiological ventricular deformation on the morphology of the t-wave: a hybrid, static-dynamic approach. IEEE Transactions on Biomedical Engineering 2011;58(7):2109-2119.

[7] Roth BJ. Electrical conductivity values used with the bidomain model of cardiac tissue. IEEE Transactions on Biomedical Engineering 1997;44(4):326-328.

Address for correspondence:

Ushenin Konstantin Sergeevich

Ural Federal University

620002, 19 Mira street, Ekaterinburg, Russia

konstantin.ushenin@urfu.ru 